\title{
Occurrence and Phenotypic Characterization of Multidrug-Resistant Bacterial Pathogens Isolated from Patients in a Public Hospital in Mogadishu, Somalia
}

\author{
Ayan Aden Moussa (iD) ${ }^{1,2}$ \\ Abdkerem Abdulahi Abdi ${ }^{1,2}$ \\ Mohamed Abdullahi Awale (iD) ${ }^{1,2}$ \\ Bashiru Garba $\left.{ }^{3}\right)^{3}$ \\ 'Institute for Medical Research, SIMAD \\ University, Mogadishu, Somalia; ${ }^{2}$ Faculty \\ of Medicine and Health Science, SIMAD \\ University, Mogadishu, Somalia; \\ ${ }^{3}$ Department of Veterinary Public Health \\ \& Preventive Medicine, Usmanu \\ Danfodiyo University, Sokoto, Sokoto \\ State, Nigeria
}

This article was published in the following Dove Press journal: Infection and Drug Resistance

Purpose: This study reports a cross-sectional investigation to determine the antimicrobial resistance pattern of the common bacterial contaminants isolated from hospitalized patients in Mogadishu, Somalia.

Materials and Methods: A total of 328 clinical samples comprising urine, blood, vaginal swab, pus aspirates, and stool were collected from a public hospital located in Mogadishu the capital city of Somalia between October 2019 to March 2020. The isolation and biochemical characterization of the bacterial isolates were performed using the conventional culture and biochemical assay tests. Similarly, antimicrobial susceptibility was determined using the Kirby-Bauer disk diffusion.

Results: A total of 275 pathogenic bacteria that include Staphylococcus aureus, Escherichia coli, Pseudomonas aeroginosa, Proteus vulgare, Klebsiella pneumonia, and Salmonella spp. were detected with an overall detection rate of $78.4 \%$ (257/328). Among the bacterial pathogens isolated from clinical specimens, 152 (46.3\%) were Staphylococcus aureus, 60 (18.3\%) were E. coli, 10 (3.1\%) Proteus vulgaris, 6 (1.8\%) Klebsiella pneumonia, and 1 $(0.3 \%)$ isolate was found to be Salmonella $\mathrm{sp}$. The antimicrobial susceptibility assay revealed variable resistance pattern with clindamycin (40\%), ampicillin (27\%), vancomycin (26\%), levofloxacin $(23 \%)$, amoxicillin $(20 \%)$, ciprofloxacin (18\%) and nitrofurantoin (12\%) showing the highest rate of resistance. Moreover, evaluation of multidrug resistance showed that Staphylococcus aureus had the highest multidrug resistance rate, with 19 isolates showing resistance to more than two drugs, followed by E. coli with three isolates. In contrast, each of Proteus vulgare, Salmonella sp. and Klebsiella pneumonia had one isolate each that exhibited multidrug resistance characteristics.

Conclusion: The findings of this study showed the occurrence of a number antimicrobialresistant bacterial pathogens whose prevalence varies with age and sex. Therefore, there is a need for comprehensive antimicrobial profiling of bacterial isolates during the management of patients in the hospital.

Keywords: nosocomial infection, antimicrobial resistance, Somalia, multidrug-resistant pathogens, hygiene, sanitation

\section{Introduction}

Staphylococcus aureus, Escherichia coli, Pseudomonas aeruginosa, Proteus mirabilis, and Klebsiella pneumonia are adjudged as the most common cause of hospital-acquired infections responsible for a wide range of clinical complications 
including pneumonia, urinary tract infections, and septicemia among others. ${ }^{1-3}$ They constitute an important group of opportunistic pathogens that survive in hospital environments contaminating floors, beds, equipment and medical devices, respiratory airways, and human skins. ${ }^{4}$ Unhygienic hospital environment and contaminated medical devices are believed to be the most common source of infection in nosocomial transmission. The pathogens can also be transmitted by health personnel during healthcare delivery, hence the need for healthcare staff to maintain good personal hygiene. ${ }^{5}$ The incidence of these healthcareassociated infections has reached $75 \%$ in sub-Saharan Africa and has been responsible for $56 \%$ of all neonatal death. ${ }^{6}$

The rise of antimicrobial resistance among pathogens causing hospital-acquired infections constitutes a serious economic and public health problem. ${ }^{7-9}$ The development of resistance follows pathogen mutations due to selective pressures from the use and misuse of antimicrobials during patient treatment. In resource-poor countries, resistant bacterial organisms have made the hospital environment a niche with a manifold of opportunities for transmission, partly because of the interplay of a high number of susceptible patients, poor healthcare delivery services, unhygienic state of patients and their relatives as well as the unregulated use of antimicrobials. ${ }^{10,11}$

The population health in Somalia is in a dire state, occasioned by famine, malnutrition, displacement as a result of the insurgency and civil unrest. In addition to the plethora of endemic illnesses such as diarrhoeal disease, measles, malaria, and acute respiratory illnesses, many of the mortalities, especially among children, are the result of infection of these antimicrobial-resistant pathogens. The infections have led to increased morbidity and mortality as well as economic hardship due to prolonged hospitalization. Although some investigations have been conducted on antimicrobial resistance among Escherichia coli in outpatients with urinary tract infections, as well as Staphylococcus aureus isolated from patients with skin wound infection, the present investigation was undertaken with a wider purview. ${ }^{12,13}$ The study was set out to investigate the prevalence of multidrugresistant pathogenic bacterial organisms from clinical samples collected from a public hospital in Mogadishu, Somalia, during a five months period. The samples (vaginal swabs, blood, stool, and urine) were collected from the patients under hospitalization, and the phenotypic characterization of drug resistance among the positive isolates was undertaken.

\section{Materials and Methods Ethics Statement}

The study was approved by the ethics committee of the SIMAD University (reference number IMRSU/FHMS (FR18) P002). All the respondents voluntarily provided informed consent for their participation after being adequately informed of the study's aim and objectives according to the Declaration of Helsinki.

\section{Study Design and Setting}

The participants for the present investigation were patients hospitalized at a specialist public-private hospital centrally located in the capital city of Mogadishu. The samples were collected using a purposive sampling technique where patients hospitalized for 48 hours or more are sampled before the commencement of antimicrobial therapy. The hospital is patronized by residents within the Mogadishu city and its neighbouring towns including Baidoa, Kismayo, and Galkayo among others. The hospital is a 72 beds capacity hospital offering comprehensive healthcare services including public enlightenment on the dangers of emerging infectious diseases in line with the Ministry of Health policies to Somalis in the past six years.

\section{Sample Population and Sample Size Determination}

This cross-sectional study was conducted at a public hospital in Mogadishu, Somalia, from October 2019 to March 2020. During this investigation, all patients under hospitalization for more than 48 hours at different wards, including the intensive care unit, surgical wards, medical wards, and pediatric wards, were sampled. A total of 328 samples comprising 231 urine samples, 56 vaginal swabs, 24 pus aspirates, 14 diarrheal specimens, and three (3) blood samples were collected.

The sample size was determined using the $26.5 \%$ prevalence of Staphylococcus aureus reported by Mohammed et al among outpatients with urinary tract infections in Somalia. $^{13}$

The formula $\mathrm{N}=\mathrm{Z}^{2} \mathrm{P}(1-\mathrm{P}) / \mathrm{e}^{2}$ was used for the calculation. $^{14}$

Where $\mathrm{Z}$ is 1.96 at $95 \%$ confidence interval; $\mathrm{P}$ is reported prevalence $(0.265)$, and e is $5 \%$ margin of error. 
Therefore

$\mathrm{N}=3.8416 \times 0.256 \times 0.735 / 0.0025=299.9$

Although 300 samples were targeted, we were able to collect 328 samples during the collection period.

\section{Sample Collection and Bacterial Identification}

After aseptically collecting the samples in sterile specimen bottles and liquid media transport swabs (Oxoid), the samples were transported to the Bacteriology Laboratory, Faculty of Medical Sciences, SIMAD University, Mogadishu Somalia in an icebox to prevent them from multiplying further. Upon arrival at the laboratory, the samples were inoculated onto tryptic soy broth and incubated at $37^{\circ} \mathrm{C}$ for 24 hours for pre-enrichment. After the 24 hour pre-enrichment, $100 \mu \mathrm{L}$ of the overnight culture was used to inoculate nutrient agar (Oxoid), blood agar (Oxoid) MacConkey agar (Oxoid) plates and incubated for $24 \mathrm{~h}$ at $37{ }^{\circ} \mathrm{C}$ and observed for bacterial growth. Grown bacterial colonies were subjected to Gram staining, and identification was made based on colonial morphological characteristics. Presumptive colonies were further evaluated for their biochemical properties including oxidase and Simons citrate test, coagulase test, catalase test, motility assay as well as SIM (hydrogen sulfide production, indole, and motility tests).

\section{Antibiotic Susceptibility Testing}

The phenotypic antimicrobial susceptibility profile of the isolates was conducted using the Kirby-Bauer's disk diffusion method according to the procedure stipulated by the Clinical Laboratory Standards Institute (CLSI) guidelines. ${ }^{15}$ The antimicrobial susceptibility assay was determined using a panel of 20 antimicrobials including; ciprofloxacin (CIP); amikacin (AK); cefotaxime (CTX); ceftazidime (CAZ); levofloxacin (LEV); trimethoprimsulfamethoxazole (SXT); meropenem (MRP); Ampicillin (AMP); ceftriaxone (CRO); cefuroxime (CXM); amoxicillin-clavulanic acid (AUG); nitrofurantoin $(\mathrm{F})$; gentamicin $(\mathrm{CN})$; nalidixic acid (NAL); vancomycin (VA); clindamycin (CD); colistin (CST); azithromycin (AZM); linezolid (LNZ); norfloxacin (NOR). In order not to overestimate the antimicrobial resistance characteristics of the isolates, the results were documented as either susceptible (which include all susceptible and intermediately susceptible isolates-S) or resistant (R). Similarly, multidrug-resistance (MDR) was determined when isolates exhibited resistance to at least three or more of the antimicrobials tested. E. coli ATCC 25922 was used as a control strain.

\section{Statistical Analysis}

The results were expressed in percentages, and statistical analysis using Chi-square to determine association between different variables (age, sex, and sample source) was done using SPSS v.25 software. Statistical significance was established when $\mathrm{p}<0.05$.

\section{Results}

A total of 328 samples comprising 152 urine samples, 56 vaginal swabs, 24 pus aspirates, 14 stool samples, and three (3) blood samples were collected from hospitalized patients at the public hospital Mogadishu, Somalia. Among all the sources sampled, 257 (78.4\%) had bacterial growth. After the isolation and biochemical characterization, 152 (46.3\%) were Staphylococcus aureus, 60 (18.3\%) were E. coli, 10 (3.1\%) Proteus vulgaris, 6 (1.8\%) Klebsiella pneumonia, and $1(0.3 \%)$ isolate was found to be Salmonella $\mathrm{sp}$.

Similarly, the distribution of the bacterial isolates according to gender showed a statistically significant association $\mathrm{X}^{2} 11.158 ; \mathrm{p}=0.04(5, \mathrm{n}=328)$ with sex where the isolates from the female patients were the highest with 152 isolates representing 59\% while the male samples had a positive growth amounting to 105 which is $40.9 \%$ of the positive isolates (Figure 1).

Furthermore, in this study, the age predisposition was classified into adult and child, and the results showed that 229 samples were collected from adult male patients with $179(69.7 \%)$ being culture positive, while a total of 99 samples were collected from children with 78 (30.4\%) being culture-positive (Figure 2). The Chi-square analysis of this relationship showed that there was a significant association between bacterial isolation and the age of the patients $\mathrm{X}^{2}$ 19.414; $\mathrm{p}=0.002(5, \mathrm{n}=328)$ where adult patients were found to be more susceptible.

Antimicrobial susceptibility test conducted for the 275 isolates against a panel of 20 different antimicrobial drugs is presented in Table 1. The study indicated that the drug resistance rates of the 275 bacterial isolates varied considerably with clindamycin (40\%), ampicillin (27\%), vancomycin (26\%), levofloxacin (23\%), amoxicillin (20\%), ciprofloxacin (18\%) and nitrofurantoin (12\%) showing the highest rate of resistance. Similarly, multidrug resistance profile of the isolates was evaluated based on resistance to three or more of the antimicrobials tested, and the results 


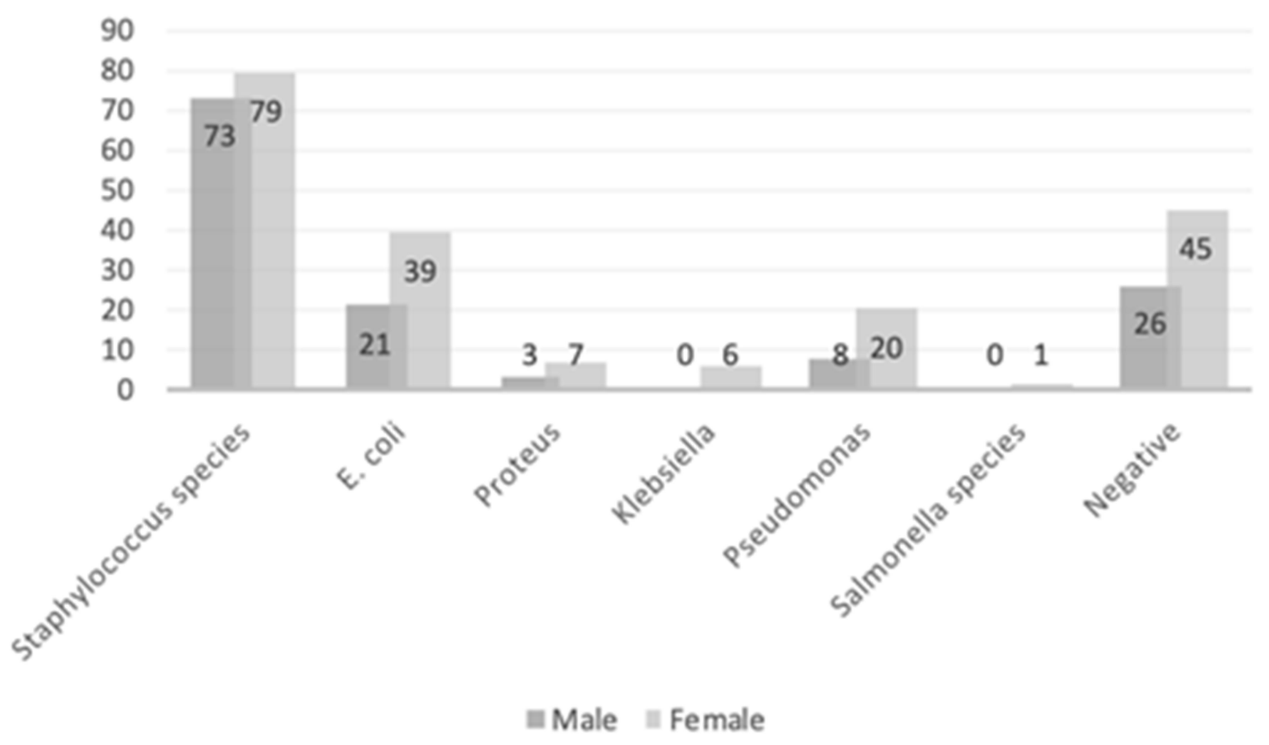

Figure I Prevalence of bacterial species isolated from positive cultures according to gender.

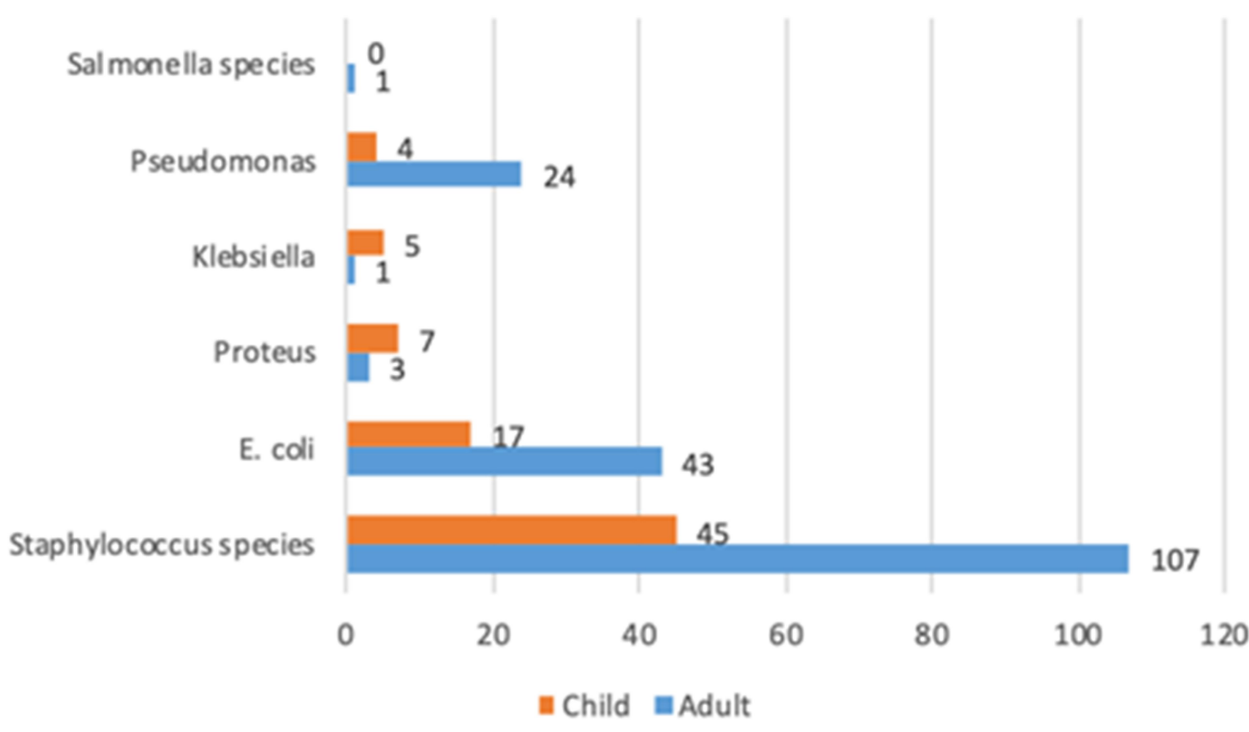

Figure 2 Illustration of bacterial growth from samples analyzed according to age category.

indicated that Staphylococcus aureus had the highest multidrug resistance rate with 19 isolates showing resistance to more than two drugs, followed by $E$. coli with three isolates while each of Proteus vulgare, Salmonella sp. and Klebsiella pneumonia had one isolate each that exhibited multidrug resistance characteristics (Figure 3).

\section{Discussion}

The overall prevalence of bacterial contamination detected in the present investigation revealed a considerably high rate of contamination (78.4\%). However, the high contamination may not be unconnected with the poor hygiene of patients and the hospital environment, which could pave the way for the increased incidence of nosocomial infections. The study also discovered Staphylococcus aureus as the most common bacterial contaminant across all the various sampling sources, followed by E. coli and P. aeruginosa. Staphylococcus aureus is one of the major pathogenic organisms inhabiting the hospital environment and has proven to be a serious public health problem following the emergence of methicillin-resistant strains of the bacteria. ${ }^{16}$ In the same vein, E. coli and 


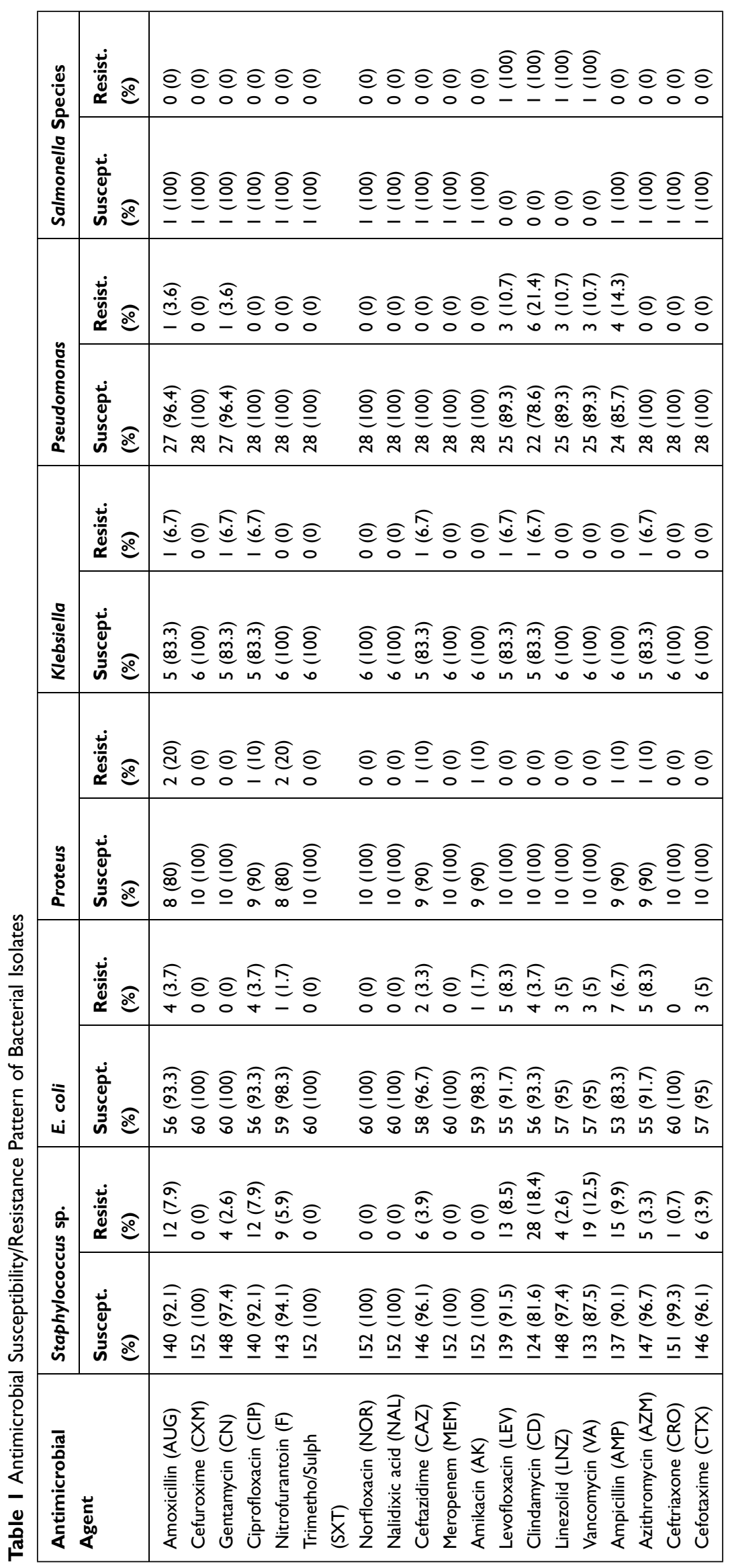




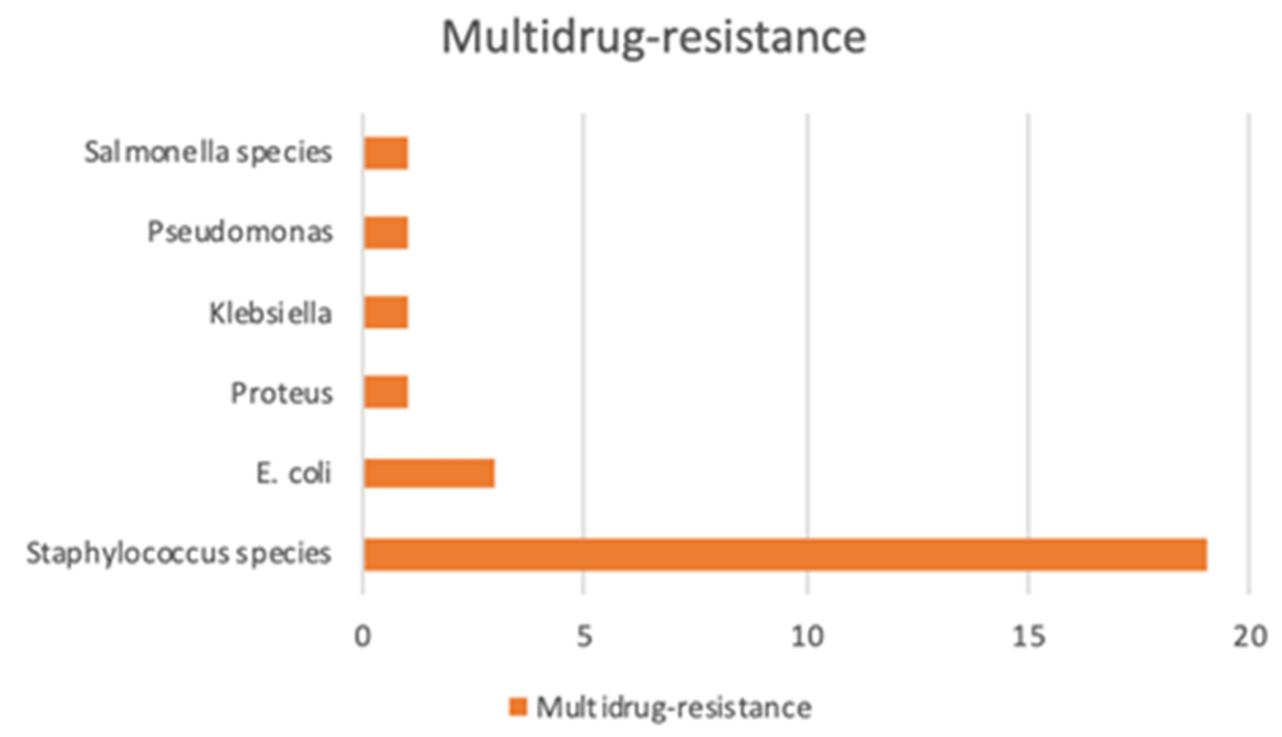

Figure 3 Distribution of multidrug-resistant bacterial isolates obtained from hospitalized patients in the hospital, Mogadishu, Somalia.

P. aeruginosa are two other very popular pathogenic bacteria commonly transmitted in the hospital settings among health workers and their patients. In recent years, these bacteria species have developed resistance to the commonly used disinfectants in hospitals, which has led to increased cases of hospital-acquired infections as a result.${ }^{17,18}$ Previous studies in Iran, Sudan, and Somalia have all reported a high rate of bacterial contamination among patients under hospitalization, which conforms with the result of the present study. ${ }^{12,13,19}$ In each of the aforementioned reports, the poor healthcare delivery services, and poverty may have played a role in the high rate of bacterial isolation observed.

The most prevalent bacterial contaminant in the present study is Staphylococcus aureus, with $46.3 \%$. This outcome is higher than the $26 \%$ reported by Hussein et al among patients with skin wound infection in Shaafi Hospital Mogadishu, Somalia. This disparity may be because they only studied 50 wound swabs, while our investigation included a large number of urine samples. Patients with urinary tract infections have been reported to harbour Staphylococcus species, which may explain the discrepancy observed in our study in relation to earlier reports in Somalia. ${ }^{20}$ Also, this investigation detected E. coli, Proteus vulgaris, and Klebsiella pneumonia in 18.3\%, $3.1 \%$, and $1.8 \%$ of the isolates, respectively. The detection of these enteric bacteria may suggest possible faecal contamination and poor personal hygiene and sanitation among patients and health workers. Moreover, a statistically significant association was observed between the isolation rate and sex as well as age. While the sex predisposition contradicts the popular notion that male is more exposed to these environmental contaminants due to individual behavioural differences including hygiene and eating practices increased tendency for men to eat raw food, as well as patronizing roadside food due to the nature of their jobs particularly labourers. ${ }^{21,22}$ The fact that female patients were found to have higher bacterial carriage may be because of their nursing responsibilities, where they frequently have to clean and change diapers when their children defecate.

This study also evaluated the antimicrobial resistance patterns of the bacterial isolates obtained from hospitalized patients. This is owing to the role of antibiotic resistance among enteric pathogens plays in causing severe, often lifethreatening infections among hospitalized patients, particularly women and children. ${ }^{7}$ Moreover, understanding the AMR profile of the commonly circulating pathogens can help physicians during prescriptions and recommendations for prevention and control strategies to be employed against these deadly bugs. The result of the present study revealed that the greater percentage of the positive cultures was susceptible to an array of antimicrobials tested, with amikacin, meropenem, nalidixic acid, norfloxacin, sulfamethazine/trimethoprim and cefuroxime being the most potent antimicrobials against all the positive isolates. However, a considerable number of resistance patterns were also observed where clindamycin (40\%), ampicillin (27\%), 
vancomycin (26\%), levofloxacin (23\%), amoxicillin (20\%), ciprofloxacin (18\%) and nitrofurantoin (12\%) exhibited the highest resistance rate. Antimicrobial resistance represents a serious health challenge globally and has been reported to cause 700,000 deaths annually. ${ }^{19}$ The increased resistance levels observed against clindamycin, ampicillin, vancomycin, levofloxacin, amoxicillin, ciprofloxacin, and nitrofurantoin is a worrying sign as they represent some of the most commonly used drugs in the management of human illnesses. The resistance observed could be attributed to the unregulated use by the general population because of the ease with which they can be purchased over the counter, even without any prescription. Furthermore, a multidrug resistance pattern was also observed among the bacterial isolates with Staphylococcus and E. coli having 19 and three isolates with resistance against Levofloxacin, Clindamycin, Vancomycin, Ampicillin, Ciprofloxacin, and Nitrofurantoin. These observations are in congruence with earlier reports where Staphylococcus aureus, E. coli and Klebsiella pneumonia were found to exhibit multidrug resistance, hence the urgent need for immediate intervention to slow down the spread of this public health threat. ${ }^{4,23}$

\section{Conclusion}

This investigation was able to establish the presence of Staphylococcus aureus, Escherichia coli, Pseudomonas aeroginosa, Klebsiella pneumonia, and Salmonella sp. as the most common bacterial contaminants in the hospital, with the potential for causing nosocomial infection. The bacterial isolates were also found to exhibit multidrug resistance characteristics, which is viewed as an important public health challenge, especially in Somalia, where the healthcare services are not adequate. Therefore, there is an urgent need for stakeholders and concerned authorities to scale up and strengthen the healthcare delivery services to curb the menace of the antimicrobial-resistant pathogens in Somalia.

\section{Data Sharing Statement}

The data supporting the conclusions of this article are included in the article.

\section{Ethics and Consent to Participate}

The study was approved by the ethics committee of the SIMAD University (reference number IMRSU/FMHS (FR18) P002). All potential respondents were contacted personally and thoroughly informed about the aim of the study, data processing, and the use of the data.
Participation was voluntary and participants could refuse to participate.

\section{Acknowledgment}

The authors acknowledge the staff and students of Institute for Medical Research, SIMAD University that helped during the conduct of the study.

\section{Funding}

This research project was supported by the Centre for Research and Development (CRD), (CRD, grant no. SUDA-RGL-2019-001). The funder had no role in study design, data collection, analysis, decision to publish, interpretation of data or preparation of the manuscript.

\section{Disclosure}

The authors report no conflicts of interest in this work.

\section{References}

1. Agaba P, Tumukunde J, Tindimwebwa JVB, Kwizera A. Nosocomial bacterial infections and their antimicrobial susceptibility patterns among patients in Ugandan intensive care units: a cross sectional study. BMC Res Notes BioMed. 2017;10:349. doi:10.1186/s13104-017-2695-5

2. Zhanel GG, DeCorby M, Laing N, et al. Antimicrobial-resistant pathogens in intensive care units in Canada: results of the Canadian National Intensive Care Unit (CAN-ICU) study, 2005-2006. Antimicrob Agents Chemother. 2008;52:1430-1437. doi:10.1128/AAC.01538-07

3. Garba B, Habibullah SA, Saidu B, Suleiman N. Effect of mastitis on some hematological and biochemical parameters of Red Sokoto goats. Vet World. 2019;12(4):12. doi:10.14202/vetworld.2019.572-577

4. Du J, Li P, Liu H, Lü D, Liang H, Dou Y. Phenotypic and Molecular characterization of multidrug resistant Klebsiella pneumoniae Isolated from a University Teaching Hospital, China. Mokrousov I, editor. PLoS One. 2014;9:e95181. doi:10.1371/journal.pone.0095181

5. Khan HA, Baig FK, Mehboob R. Nosocomial infections: epidemiology, prevention, control and surveillance. Asian Pac J Trop Biomed. 2017;7(5):478-482. doi:10.1016/j.apjtb.2017.01.019

6. WHO. The Burden of Health Care-Associated Infection Worldwide. WHO World Health Organization; 2013.

7. Folgori L, Bielicki J, Heath PT, Sharland M. Antimicrobial-resistant Gram-negative infections in neonates: burden of disease and challenges in treatment. In: Current Opinion in Infectious Diseases. Lippincott Williams and Wilkins;2017:281-288. Available from: https://pubmed.ncbi.nlm.nih.gov/28306563/. Accessed July 13, 2020.

8. Salihu M, Garba B, Isah Y. Sokoto Journal of Veterinary Sciences Evaluation of microbial contents of table eggs at retail outlets in Sokoto metropolis, Nigeria. Sokoto J Vet Sci J Vet Sci. 2015;13:22-28.

9. Zakaria Z, Hassan L, Sharif Z, et al. Analysis of Salmonella enterica serovar Enteritidis isolates from chickens and chicken meat products in Malaysia using PFGE, and MLST. BMC Vet Res. 2020;16(1):16. doi:10.1186/s12917-020-02605-y

10. Wieler LH, Ewers C, Guenther S, Walther B, Lübke-Becker A. Methicillin-resistant staphylococci (MRS) and extended-spectrum beta-lactamases (ESBL)-producing Enterobacteriaceae in companion animals: nosocomial infections as one reason for the rising prevalence of these potential zoonotic pathogens in clinical samples. Int $J$ Med Microbiol. 2011;301(8):635-641. doi:10.1016/j. ijmm.2011.09.009 
11. Garba B, Bahaman AR, Zakaria Z, et al. Antigenic potential of a recombinant polyvalent DNA vaccine against pathogenic leptospiral infection. Microb Pathog. 2018;124:124. doi:10.1016/j. micpath.2018.08.028

12. Hussein AS, Hirsi HA, Dirie FA, Osman FM, Hassan SA Antimicrobial Susceptibility of Staphylococcus aureus isolated from patient with skin wound infection in Shaafi Hospital-MogadishuSomalia. 2018. Available from: www.usa-journals.com., accessed on July 13, 2020.

13. Mohamed MA, Abdifetah O, Hussein FA, Karie SA. Antibiotic resistance pattern of Escherichia coli isolates from outpatients with urinary tract infections in Somalia. J Infect Dev Countries. 2020;14:284-289. doi:10.3855/jidc.12189

14. Thrusfield M. Veterinary epidemiology. In: Third Edit. Preventive Veterinary Medicine. Blackwell Publishing Ltd; 2008:233-236 p.

15. CLSI. Performance Standards for Antimicrobial Susceptibility Testing an Informational Supplement for Global Application Developed Through the Clinical and Laboratory Standards Institute Consensus Process. 26th ed. Wayne: PA 19087 USA; 2016.

16. Solberg CO. Spread of Staphylococcus aureus in hospitals: causes and prevention. Scandinavian J Infect Dis. 2000;32(6):587-595. doi:10.1080/003655400459478

17. Toval F, Köhler CD, Vogel U, et al. Characterization of Escherichia coli isolates from hospital inpatients or outpatients with urinary tract infection. J Clin Microbiol Am Soc. 2014;52:407-418. doi:10.1128/ JCM.02069-13
18. Messina G, Ceriale E, Lenzi D, Burgassi S, Azzolini E, Manzi P. Environmental contaminants in hospital settings and progress in disinfecting techniques. Biomed Res Int. 2013;2013:1-8. doi:10.1155/ 2013/429780

19. Azimi T, Maham S, Fallah F, Azimi L, Gholinejad Z. Evaluating the antimicrobial resistance patterns among major bacterial pathogens isolated from clinical specimens taken from patients in Mofid Children's Hospital, Tehran, Iran: 2013-2018. Infect Drug Resist. 2019;12:2089-2102. doi:10.2147/IDR.S215329

20. Lafon T, Hernandez Padilla AC, Baisse A, et al. Community-acquired Staphylococcus aureus bacteriuria: a warning microbiological marker for infective endocarditis? BMC Infect Dis BioMed Central Ltd. 2019;19:504. doi:10.1186/s12879-019-4106-0

21. Khan M. A plausible explanation for male dominance in typhoid ileal perforation. Clin Exp Gastroenterol. 2012;5:213-217. doi:10.2147/ CEG.S36569

22. Vázquez-Martínez ER, García-Gómez E, Camacho-Arroyo I, González-Pedrajo B. Sexual dimorphism in bacterial infections. Biol Sex Differ. 2018 Dec;9(1):1-20.

23. Sserwadda I, Lukenge M, Mwambi B, Mboowa G, Walusimbi A, Segujja F. Microbial contaminants isolated from items and work surfaces in the post- operative ward at Kawolo general hospital, Uganda. BMC Infect Dis BioMed Central Ltd. 2018;18:68. doi:10.1186/s12879-018-2980-5
Infection and Drug Resistance

\section{Publish your work in this journal}

Infection and Drug Resistance is an international, peer-reviewed openaccess journal that focuses on the optimal treatment of infection (bacterial, fungal and viral) and the development and institution of preventive strategies to minimize the development and spread of resistance. The journal is specifically concerned with the epidemiology of

\section{Dovepress}

antibiotic resistance and the mechanisms of resistance development and diffusion in both hospitals and the community. The manuscript management system is completely online and includes a very quick and fair peerreview system, which is all easy to use. Visit http://www.dovepress.com/ testimonials.php to read real quotes from published authors. 